\title{
0231 SERUM S100-B AS A TOOL TO PREDICT COMPUTED CRANIAL TOMOGRAPHY FINDINGS IN MILD BRAIN INJURY
}

D Zongo*, R Ribereau-Gayon, F Masson, E Lagarde Correspondence: PPCT (Prevention et prise en charge des traumatismes) ISPED-Bordeaux, INSERM U897. Case 11 Universite Victor Sgalen Bordeaux 2146 rue Leo Saignat 33076 BORDEAUX 33076, France

\subsection{6/ip.2010.029215.231}

Background Minor head injury (MHI) has a high incidence and implies a risk of brain injury. Cranial computed tomography (CCT) is recommended for early detection of these lesions. However the indication of CCT is based on non specific clinical symptoms. Protein S100-B was then suggested as a promising tool to identify those patients with no risk of cranial injury.

Objective To evaluate S100-B as a screening tool in the management of $\mathrm{MHI}$.

Methods Patients attending Bordeaux emergency department with an isolated MHI were consecutively included during 15 months. Blood samples were drawn within $6 \mathrm{~h}$ after head injury to assess S100-B level.

Results 1559 patients were included. CCT was indicative of cranial injury in 112 patients (7\%). At the threshold of $0.10 \mathrm{~g} / \mathrm{l}, \mathrm{S} 100 \mathrm{~B}$ has a sensitivity of $99 \%$ (95\% CI 95 to 100$)$, 


\section{IP Safety 2010 abstracts}

a specificity of $12 \%(11-14)$ and a negative predictive value (NPV) of $99 \%$ (97-100) as compared with CCT results. $12 \%$ of the patients has a S110-B level below this threshold and therefore could have been discharged with CCT. A threshold of $0.12 \mu \mathrm{g} / \mathrm{L}$ proved however to be the best compromise between sensitivity (99\%), specificity (20\%) and a NPV of $99.7 \%$ (98-100) with CCT potentially avoided for $19 \%$ of the patients.

Discussion Early determination of S100-B blood levels is highly sensitive to detect CCT abnormalities. The threshold of $0.12 \mu \mathrm{g} / \mathrm{L}$ was the best compromise between sensitivity and specificity in our study. 